\title{
Design and Fabrication of Self Balancing Two Wheeler Vehicle Using Gyroscope
}

\author{
Pallav Gogoi ${ }^{\# 1}$, Manish Nath ${ }^{\# 2}$, Bumi Trueman Doley ${ }^{\# 3}$, Abhijit Boruah ${ }^{\# 4}$, Hirok Jan Barman ${ }^{\# 5}$

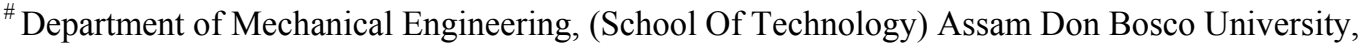 \\ Azara Campus, Airport Road, Azara, Guwahati - 781017 \\ Assam - INDIA \\ 1 gpallav06@gmail.com \\ 2 manish4741@gmail.com \\ ${ }^{3}$ bumi.trueman@gmail.com \\ 4 abhijeet4781@gmail.com \\ 5bhirok35@gmail.com
}

\begin{abstract}
Stabilization of a two wheeled vehicle plays a vital role in the complex transportation system. Gyroscopes can deliver a major contribution towards stabilization of two wheeler vehicle. It has been speculated that gyroscopically stabilized vehicles would have higher safety with respect to normal two wheelers. The dynamic stabilization of a two-wheeled vehicle requires that a torque acting on the vehicle naturally be neutralized by a torque produced within the vehicle by a gyroscope. The gyroscope here is used as an actuator, not a sensor, by using precession forces generated by the gyroscope. When torque is applied to an axis normal to the spin axis, causing the gyroscope to precess, a moment is produced about a third axis, orthogonal to both the torque and spin axes. As the vehicle tilts from vertical, a precessioninducing torque is applied to the gyroscope assembly and the opposing gyroscopic reaction moment will tend to correct the vehicle. The key idea is that motion of the gyroscope relative to the body is actively controlled in order to generate a stabilizing moment.

We designed and fabricated a two-wheeler with self-balancing mechanism. The model works on the principle of inverted pendulum. The construction process includes the designing of the model in software, fabricating the model, assembling the model and testing. Battery is used as a power source.
\end{abstract}

Keyword- Self balancing mechanism, gyroscope, precession axis, inverted pendulum.

\section{INTRODUCTION}

There are various types of parallel spin axis wheeled two-wheeler vehicles. They are typically used for commuting or for pleasure. Lighter vehicles with smaller engines are usually cheaper than their heavier counterparts and is the primary means of transport in a lot of Asian countries. Nowadays maximum road accidents are of the two wheelers. Needless to say, a lot of investment goes into manufacturing and development of state-of the-art high technology motor bikes but none can guarantee road safety and it solely depends upon the rider and hence road accidents occur as the person riding the bike loses control over it and the bike falls. Also while learning to ride bicycles children are often afraid to ride as there is a fear of falling.

To avoid such tragic scenarios, a mechanical gyroscope can be installed in the vehicles. It works on the principle of inverted pendulum and employs the use of electromechanical components which can be used as a means of transportation for a single person. The two-wheeled, self-balancing vehicle is a non-linear multivariable and naturally an unstable system. Controlling such a system is a hard task and thus it is the topic of research. It will move forward if the user tilts in forward direction and backward if the user tilts in backward direction.

This paper presents a vehicle in which all components (mechanical, electrical) have been designed from ground up, produced, coupled together and tested. This vehicle can be viewed as ecological, battery operated and very easy to be used as system.

\section{GYROSCOPE}

A gyroscope is a spinning wheel or disc in which the axis of rotation is free to assume any orientation by itself. While rotating, the orientation of this axis is unaffected by tilting or rotation of the mounting, according to the conservation of angular momentum. Because of this, gyroscopes are useful for measuring or maintaining orientation. Gyroscopes are also based on other operating principles, such as the electronic, microchip-packaged MEMS gyroscopes found in consumer electronics devices, solid-state ring lasers, fiber optic gyroscopes, and the extremely sensitive quantum gyroscope. Applications of gyroscopes include inertial navigation systems where 
magnetic compasses would not work (as in the Hubble telescope) or would not be precise enough (as in intercontinental ballistic missiles), or for the stabilization of flying vehicles like radio-controlled helicopters or unmanned aerial vehicles, and recreational boats and commercial ships. Due to their precision, gyroscopes are also used in gyro theodolites to maintain direction in tunnel mining. Gyroscopes can be used to construct gyrocompasses, which complement or replace magnetic compasses (in ships, aircraft and spacecraft, vehicles in general), to assist in stability (Hubble Space Telescope, bicycles, motorcycles, and ships) or be used as part of an inertial guidance system.

\section{A. Gyroscope Principles}

Rigidity is the ability of a freely rotating mass to maintain its plane of spin when any external force is applied to it.

1) First Law of Gyroscope: If a rotating wheel is so maintained as to be free to move about any axis passing through its center of mass, its spin axis will remain fixed in space.

2) Second Law Of Gyroscopes: When a torque acts on a spinning mass with an axis perpendicular to that of spin, then the latter will precess about an axis perpendicular to both aforementioned axes, at an angular velocity, $\Omega,=\mathrm{T} / \mathrm{I} \omega$.

\section{B. Gyroscopic Effect}

The gyroscopic effect is widely used in air planes and ships, where in always external disturbing couple is acting on the vehicle. Thus, for the stability of such vehicles it is essential to neutralize the effect of external disturbing couple which can be done by applying equal and opposite couple. Accordingly, to generate equal and opposite reactive couple it is essential to vary the magnitude and direction of velocity of precession.

The active gyroscopic couple represents rate of change of angular momentum, and this couple must be applied to disc across the axis of spin to cause it to precess in the horizontal plane. When the axis of spin precess itself or is made to precess the shaft on which the disc is mounted applies reactive gyroscopic couple. This reactive gyroscopic couple thus produced by the gyroscope is equal to the external disturbance but it is in opposite direction. Thus this couple neutralizes the effect of disturbance and stabilizes the object.

(Active couple $)=-($ Reactive couple $)$

\section{MODEL FABRICATION}

The objective of this paper is to prove the fact that the a body can be balanced only on two parallel spin axis wheels in idle or running condition with the use of a mechanical gyroscope. The gyroscopic principle can be successfully used for creation of a prototype of a two-wheeled vehicle, and thus observing gyroscopic phenomenon on the same.

The prototype was created using following parts:

1. Steel Frame

2. Steel Base

3. $12 \mathrm{~V}$ DC Motor

4. Mild steel disc

5. Flat head stud

6. Rubber Wheels

7. Nuts and bolts (M3, M5)

8. Hub

9. Circlips

10. Ball bearings 


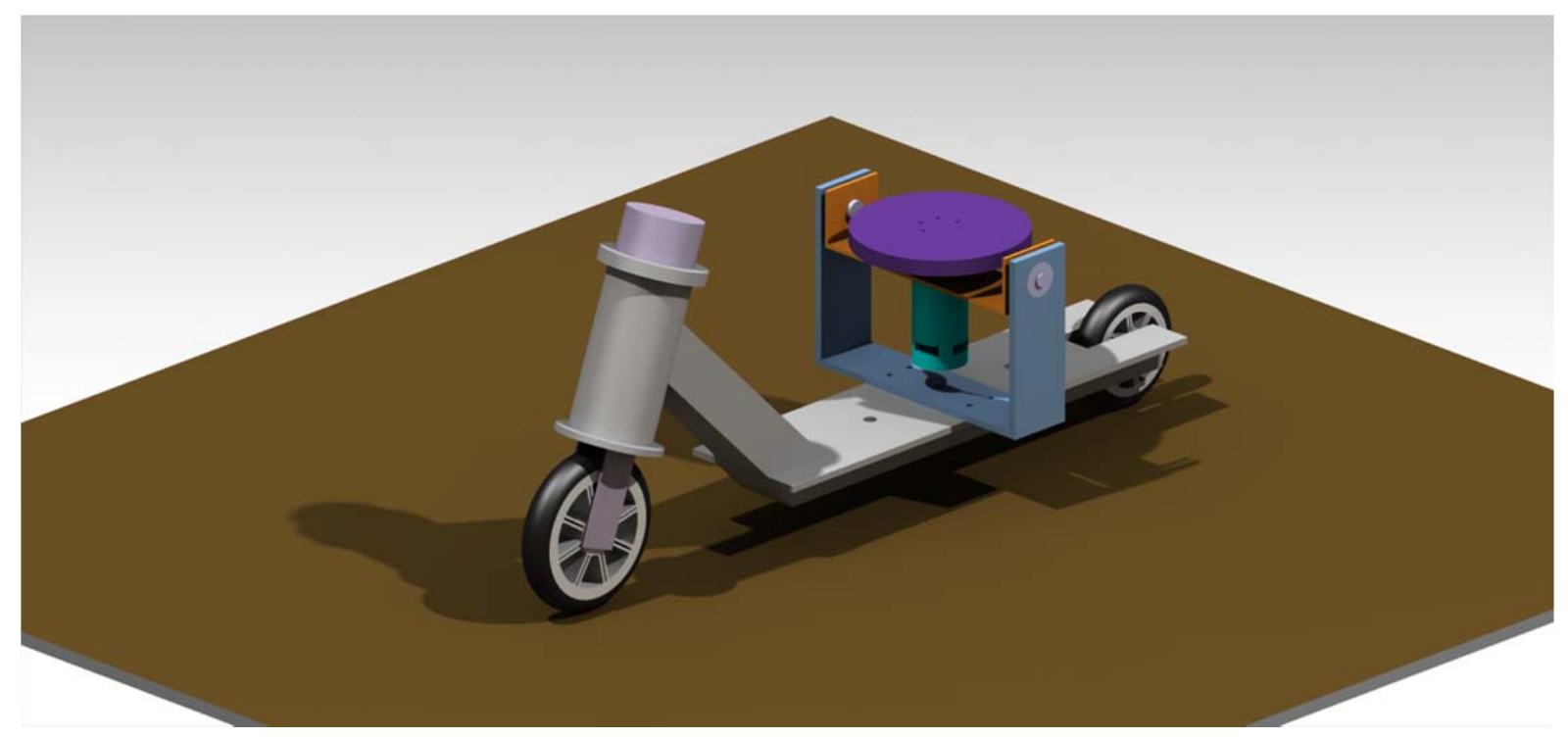

Fig.1: 3-D model of the self-balancing mechanism on a two wheeler

\section{CONSTRUCTION}

The gyroscope disc is manufactured using CNC lathe and drilling operations. The steel frame used is bolted on the bottom steel base. Round edge wheels are fitted to the steel base at the bottom, the holes in the frame are drilled in order to fit the gimble of the gyroscope assembly, the DC motor supported on a U bracket is placed on the gimble. The only pre-requisite for this setup to work is that the mass distribution, the mass should be dominant on the upper side (setup should be top heavy) of the gimble. The center of gravity is thus just above the gimble axis, stainless steel disc used as gyroscope are fitted to the shaft of the motor. The DC motor is bolted to the U-bracket due to which it remains intact with it and the shaft of the DC motor is fixed to a steel hub which has got holes drilled on its top flat surface to be used to finally fix the gyroscope disc by bolting it with the hub. The material used in making the gyroscope disc, hub, steel frame, and U-bracket is Mild Steel. To finally assemble the entire model, various sizes of nuts and bolts were used. One important design consideration that we made in this model is that the gyroscope disc should be freely suspended in the U-bracket connected to the steel frame. So for that, we used ball bearings and studs to make the angular movements and adjustments free and swift. The circlips are placed on the inner ends of the studs to avoid the studs to move out of the ball bearings, thus avoiding breakdown of the model during the running condition. The model has been made in such a way that the front wheel can move to take turns in order to change the direction of movement.

\section{WORKING}

The model is powered by a power supply unit of $12 \mathrm{~V}$ output. Once the motor starts rotating, the Mild steel disc fitted on the motor shaft starts to rotate and gradually gains speed. This rotation of the disc leads to the production of the gyroscopic effect thus, when the wheels lose their balance due to the active gyroscopic couple, a counter acting reactive gyroscopic couple is produced in the opposite direction due to gyroscopic effect, thus stabilizing the prototype model. This gyroscopic effect occurs on both left as well as right hand side. Thus, due to rotation of the gyroscope, a counter-acting reactive gyroscopic couple leads to the stabilization of the prototype. The motor and gimble axle assembly is designed in such a way that it is top heavy. This means that the center of gravity lies above the gimble axle. So the motor and gyroscope assembly tries to attain the position such that the center of gravity of the core will move downwards. But at the same time the motor and gimble assembly is arranged within the frame having bearing reaction at ends. So, the only possible way for motor to attain the stability is to either lean forward or backward. So, when the motor is started the body is about to fall on either side and also the motor assembly is leaning this causes the precession of spin axis. Due to this precession, according to right hand rule the reactive gyroscopic couple acts on the frame which nullifies the effect of the disturbing couple and thus stabilizes the vehicle. After few rotations and oscillations of motor, the motor and frame attains the stationary position and gyroscope is subjected to pure rolling motion about the spin axis 


\section{EXPERIMENTAL RESULTS}

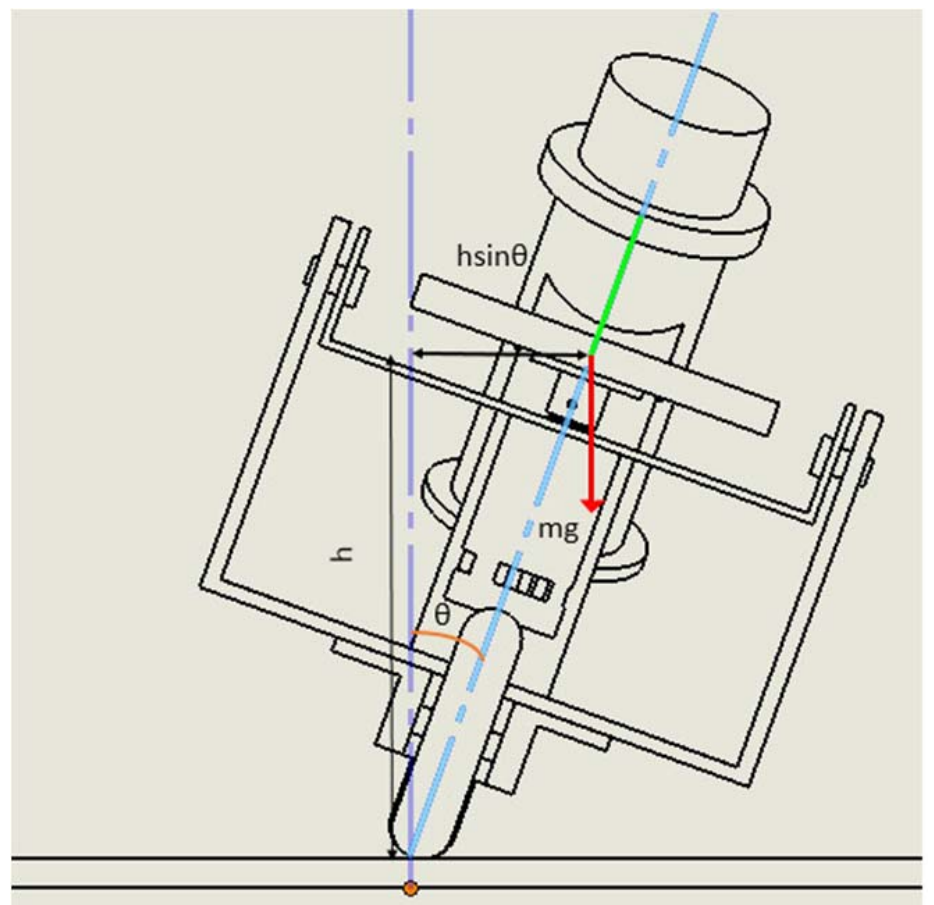

Fig.2: Sketch showing the parameters when the vehicle is leaning

Basic formulas used in calculation:

Moment of Inertia, $\mathrm{I}=\frac{m r^{2}}{2}$

Angular Momentum $=\mathrm{I} \omega=\frac{m r^{2} \pi N}{60}$

Torque, $\tau=$ Frsin $\theta$

Force, $\mathrm{F}=\mathrm{mg}$

Tilt distance $=\mathrm{h} \sin \theta$

$\mathrm{m}=$ Mass of the disc $=1.2 \mathrm{~kg}$

$\mathrm{r}=$ Radius of the disc

$\mathrm{N}=\mathrm{RPM}$

Table I

Changes of moment of inertia and angular momentum with respect to radius

\begin{tabular}{|c|c|c|c|}
\hline Serial No. & $\begin{array}{l}\text { Radius( } \\
\mathbf{m})\end{array}$ & Moment of Inertia $\left(\mathbf{k g m}^{\mathbf{2}}\right)$ & Angular Momentum(kgm $\mathbf{~} / \mathbf{s})$ \\
\hline 1 & 0.01 & 0.0001 & 0.159 \\
\hline 2 & 0.02 & 0.0004 & 0.638 \\
\hline 3 & 0.03 & 0.001 & 10436 \\
\hline 4 & 0.04 & 0.0019 & 2.553 \\
\hline 5 & 0.05 & 0.003 & 3.989 \\
\hline 6 & 0.06 & 0.004 & 5.745 \\
\hline 7 & 0.0725 & 0.0063 & 8.388 \\
\hline 8 & 0.08 & 0.007 & 10.213 \\
\hline
\end{tabular}




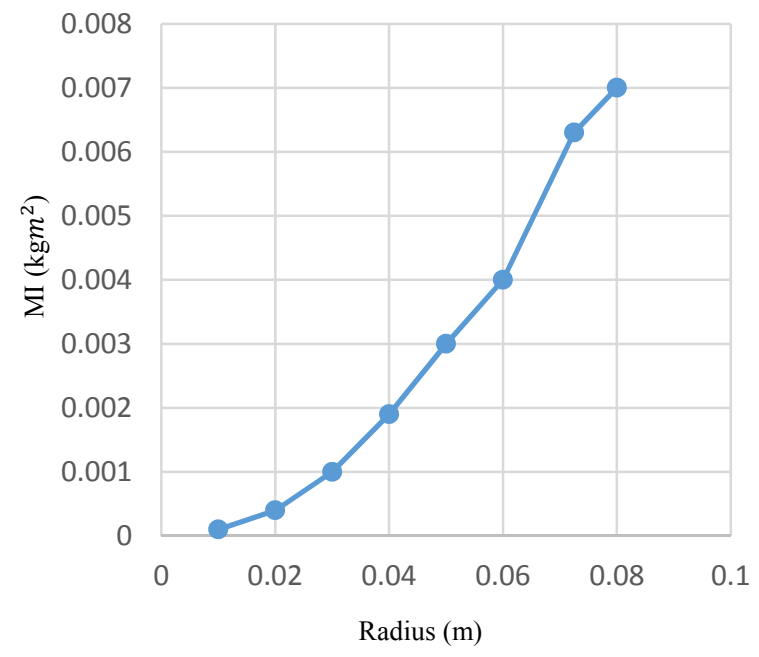

Graph 1: Radius vs Moment of Inertia, Mass-1.2kg

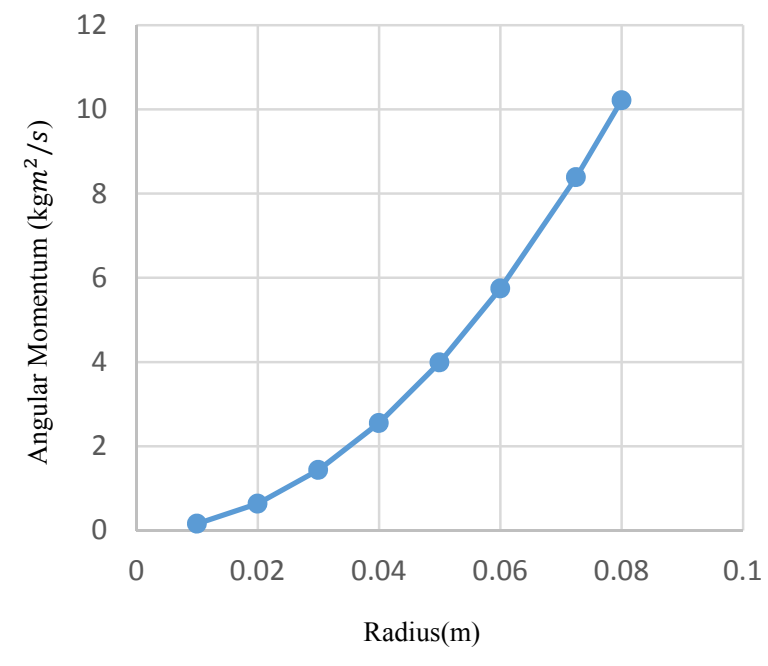

Graph 2: Radius vs Angular Momentum, Mass-1.2kg

Graph 1 gives us the clear idea that when we try to increase the radius of the gyro disc, the Moment of Inertia (MI) produced by the gyroscope increases. So, we can say that with varying radius of the gyro disc, the Moment of Inertia (MI) varies accordingly.

Graph 2 shows the relation between the radius of the gyro disc and the Angular Momentum produced by the gyroscope. Here, we can see that when we try to increase the radius of the gyro disc, the Angular Momentum also increases in response to the varying radius and vice versa which means that radius is a varying factor to obtain varying Angular Momentum values for the gyroscope.

In Graph 1 and Graph 2, we recorded the readings by keeping radius as the parameter of change to gain different readings of Moment of Inertia and Angular Momentum produced by the gyroscope respectively.

Table 2:

Changes of Tilt Distance and Torque with respect to Precession Angle

\begin{tabular}{|c|c|c|c|}
\hline Serial No. & Precession Angle( $\boldsymbol{\theta})$ & Tilt Distance(m) & Torque(Nm) \\
\hline 1 & 5 & 0.0871 & 1.0249 \\
\hline 2 & 10 & 0.173 & 2.042 \\
\hline 3 & 20 & 0.342 & 4.022 \\
\hline 4 & 30 & 0.5 & 5.88 \\
\hline 5 & 40 & 0.642 & 7.559 \\
\hline 6 & 45 & 0.707 & 8.315 \\
\hline 7 & 46 & 0.719 & 8.459 \\
\hline
\end{tabular}

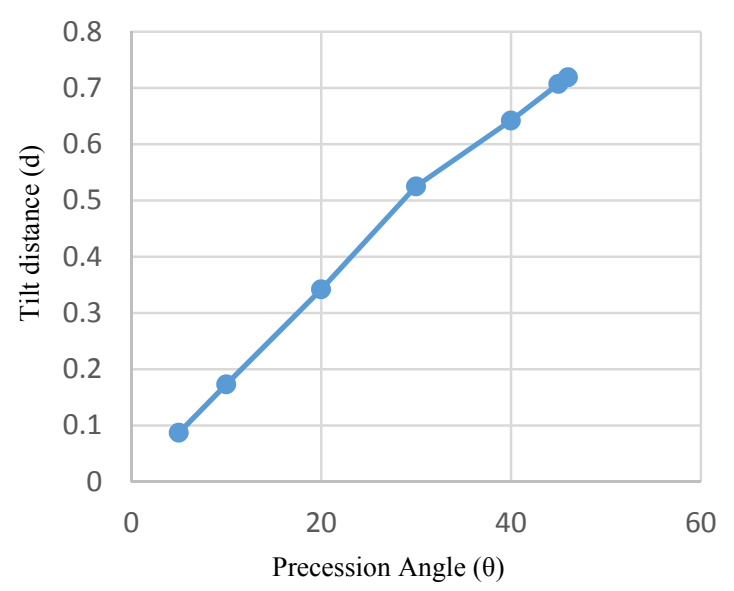

Graph 3: Precession angle vs Tilt distance, Mass1.2kg

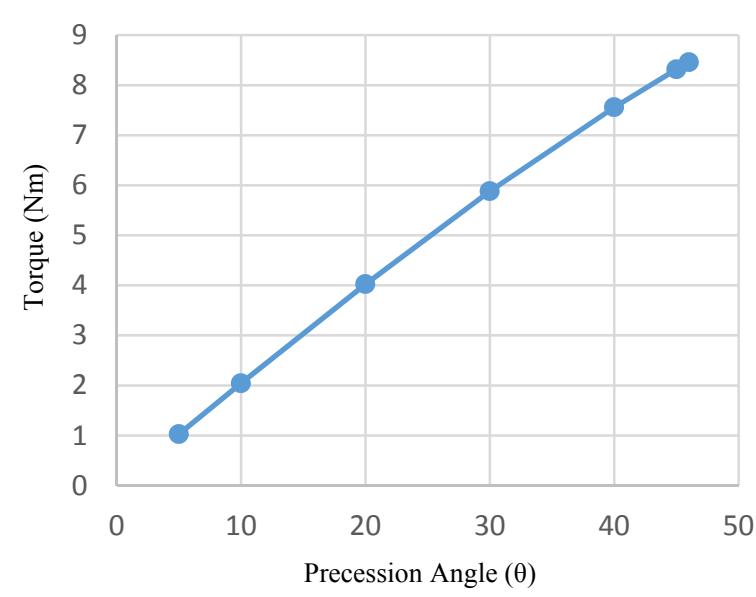

Graph 4: Precession Angle vs Torque, Mass-1.2kg

Graph 3 shows the relation between Precession angle and Tilt distance of the gyroscope. Here, if we vary the angle of precession axis, the distance of the precession axis from the normal varies accordingly. So, we can see that with increase in precession angle, the tilt distance also increases accordingly. 
Graph 4 is the representation of the results by varying the Precession angle in order to obtain corresponding values of Torque to the change in the values of Precession angle. When the Precession angle increases, we see a change in the value of Torque generated by the gyroscope. From the above graph, we can see that increasing values of Precession angle leads to increase in the Torque generated by the gyroscope.

For Graph 3 and Graph 4, we kept the Precession angle as the parameter of change which resulted in the change of Tilt distance of the Precession axis and Torque generated by the gyroscope respectively.

Table 3:

Mass vs Speed table.

\begin{tabular}{|c|c|c|}
\hline Serial No. & Mass(g) & Speed of the disc(rpm) \\
\hline 1 & 1200 & 12700 \\
\hline 2 & 1100 & 13550 \\
\hline 3 & 1000 & 14860 \\
\hline 4 & 900 & 15790 \\
\hline 5 & 800 & 16650 \\
\hline
\end{tabular}

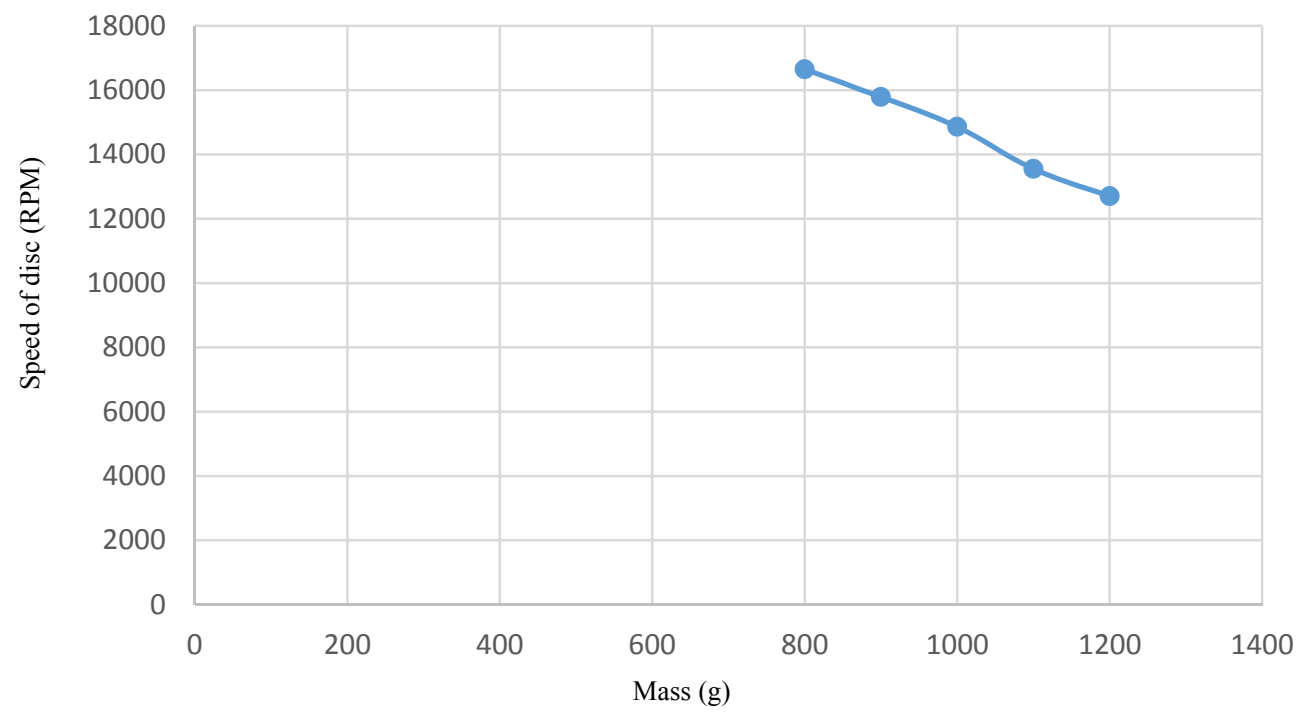

Graph 5: Mass (g) vs Speed of Disc (RPM)

Graph 5 shows the impact of Mass on the speed of the rotation of the Gyro disc. We got to know from the above graphical representation that when mass of the Gyro disc is increased, the speed of the Gyro disc decreases accordingly. The line on the graph depicts the points in relation of both speed of disc and mass when the vehicle is balanced.

\section{VII: APPLICATIONS}

The above gyroscopic stabilization concept can be used in motor bikes for advanced stability and safety and also in bicycles and other such vehicles for safer transportation. The gyroscope assembly can be placed at specific locations in the vehicle to get the stabilizing effect. In addition to being used in compasses, aircrafts, computer pointing devices, missiles etc., gyroscopes have been introduced into consumer electronics. Since the gyroscope allows the calculation of orientation and rotation, designers have incorporated them into modern technology. The integration of the gyroscope has allowed for more accurate recognition of movement within a 3D space than the previous lone accelerometer within a number of smartphones. Gyroscopes in consumer electronics are frequently combined with accelerometers (acceleration sensors) for more robust direction- and motion-sensing. Examples of such applications include smartphones, game console peripherals, and virtual reality sets. Cruise ships use gyroscopes to level motion-sensitive devices such as self-leveling pool tables. An electric powered flywheel gyroscope inserted in a bicycle wheel is being sold as a training wheel alternative.

\section{VIII: CONCLUSION}

The final model of the self-balancing vehicle design is shown below. This design has been tested at different RPMs of the disc and also with different weights to see that the vehicle is balancing. This paper presents design and fabrication of the two-wheeler self-balancing vehicle which is capable of balancing itself under application of external forces and loads. The vehicle balances itself under various conditions like forced tilt of the vehicle. Thus the proposed system can be much helpful for two-wheeled vehicles reducing accidents or unwanted falls 
and increasing safety to the rider. This system reduces the work of humans as well as provides eco-friendly environment.

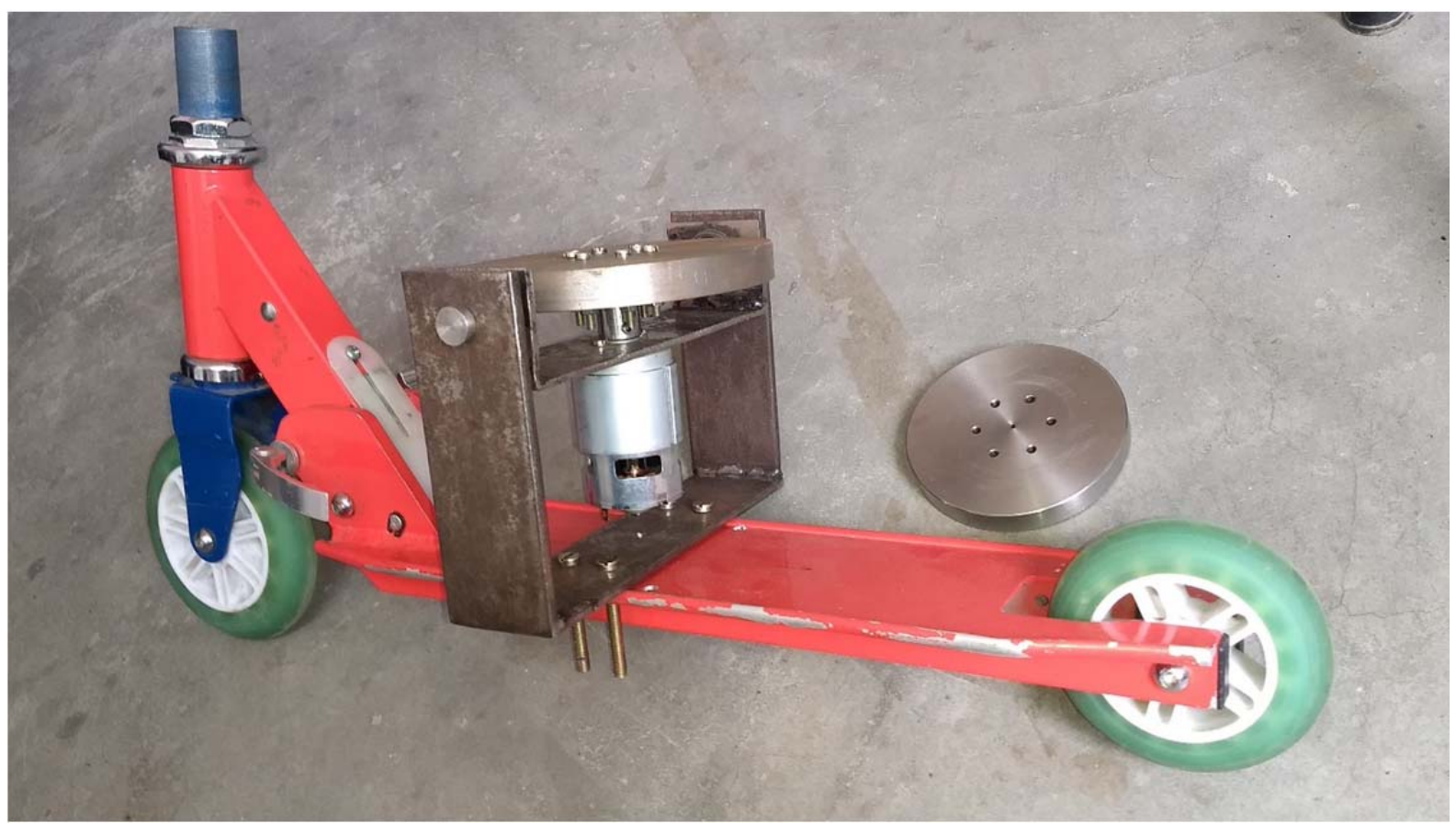

Fig.3: Final model of the design

\section{ACKNOWLEDGEMENT}

It gives us immense pleasure and satisfaction to successfully complete our endeavor in designing and fabricating a self-balancing vehicle using gyroscope. However our endeavor stands incomplete without expressing our gratitude to everyone who has contributed a lot towards the successful completion of our project work. We express our sincere thanks to Assam Don Bosco University (School of Technology) for providing us the space required to carry out our research work. We would also like to thank Micro Small and Medium Enterprise (MSME) Tool Room, Amingoan, Guwahati, Assam for providing us the machinery support necessary to make the gyroscope parts required in our project.

\section{REFERENCES}

[1] Ching-Chih Tsai, Hsu-Chih Huang and Shui-Chun Lin, "AdaptiveNeural Network Control of a Self-Balancing Two-Wheeled Scooter," IEEE transactions on industrial electronics, vol. 57, no. 4, april 2010

[2] Pierpaolo De Filippi, Mara Tanelli, Matteo Corno, Sergio M. Savaresi, and Mario D. Santucci. "Electronic Stability Control for Powered Two-Wheelers", IEEE transactions on control systems technology, vol. 22, no. 1, january 2014

[3] Karthik, Ashraf, Asif Mustafa Baig And Akshay Rao, "Self Balancing Personal Transpoter" 4th Student Conference on Research and Development, pp. 180-183, June.2006.

[4] Miseon Han, KyungHwan Kim, DoYoun Kim and Jaesung Lee, "Implementation of Unicycle Segway Using Unscented Kalman Filter in LQR control", 2013 10th International Conference on Ubiquitous Robots and Ambient Intelligence (URAI), October 31November 2, 2013 / Ramada Plaza Jeju Hotel, Jeju, Korea.

[5] Osama Jamil, Mohsin Jamil, Yasar Ayaz, Khubab Ahmad, "Modeling, Control of a Two-Wheeled Self-Balancing Robot", 2014 International Conference on Robotics and Emerging Allied Technologies in Engineering (iCREATE), Islamabad, Pakistan, April 2224, 2014

[6] Pawel Bethke,Rafal Dlugosz and Tomasz Talaska, " Project and realization of a two wheels balancing vehicle", 20th International Conference "Mixed Design of Integrated Circuits and Systems", June 20-22, 2013, Gdynia, Poland.

[7] M. A. Johnson, and M. H. Moradi, "PID Control: New Identification and Design Methods". Springer, 2005.

[8] Abdalkarim M. Mohtasib 1, and Mohsen H. Shawar, "Self-balancing Two-wheel Electric Vehicle (STEVE)", Proceedings of the 9th International Symposium on Mechatronics and its Applications (ISMA13), Amman, Jordan, April 9-11, 2013

[9] H. Ha et al., "A robust control of mobile inverted pendulum using single accelerometer," Pusan National University, Korea, The Fifteenth International Symposium on Artificial Life and Robotics, Feb, 2010.

[10] Segway Inc. (2011). "How the Segway PT Works". New Hampshire, USA. [Online] Available: http://www.segway.com/individual/ learn-how-works.php

[11] Akshay Khot, Nishad Kumbhojkar, "MODELING AND VALIDATION OF PROTOTYPE OF SELF STABILIZING TWO WHEELER USING GYROSCOPE", Department of Mechanical Engineering, Sinhgad College of Engineering, Pune, Maharashtra, India

[12] Stephen C. Spry and Anouck R. Girard, "Gyroscopic Stabilization of Unstable Vehicles: Configurations, Dynamics, and Control" March 31, 2008

[13] Simos Evangelou "THE CONTROL AND STABILITY ANALYSIS OF TWO WHEELED ROAD VEHICLES" University of London, Electrical and Electronic Engineering Imperial College London September 2003 
[14] Kealeboga Mokonopi, "BALANCING A TWO WHEELED ROBOT", University of Southern Queensland Faculty of Engineering and Surveying Bachelor of Engineering and Bachelor of Business (Mechatronics and Operations management) Submitted: November, 2006

[15] Wei An and Yangmin Li "Simulation and Control of a Two-wheeled Self-balancing Robot" IEEE, International Conference on Robotics and Biomimetics (ROBIO) Shenzhen, China, December 2013

\section{AUTHOR PROFILE}

Pallav Gogoi: Assistant Professor, Department Of Mechanical Engineering, (School Of Technology) Assam Don Bosco University, Azara, Assam-781017

Manish Nath: B. Tech. 2017 passout, Department Of Mechanical Engineering, (School Of Technology) Assam Don Bosco University, Azara, Assam-781017

Bumi Trueman Doley: B. Tech. 2017 passout, Department Of Mechanical Engineering, (School Of Technology) Assam Don Bosco University, Azara, Assam-781017

Abhijit Boruah: B. Tech. 2017 passout, Department Of Mechanical Engineering, (School Of Technology) Assam Don Bosco University, Azara, Assam-781017

Hirok Jan Barman: B. Tech. 2017 passout, Department Of Mechanical Engineering, (School Of Technology) Assam Don Bosco University, Azara, Assam-781017 